\title{
Diffuse meningo-encefalitys due to Nocardia farcinica in a young kidney transplant recipient: identification of the strain using sequencing of hsp65 gene
}

Danila Costa, Paolo Maggi', Rosa Anna Cifarelli², Giuseppe Lauria², Rosa Bruno, Laura Spinelli, Fabio Signorile', Caterina Colonna ${ }^{3}$, Giuseppe Pastore', Michele Quarto

Laboratorio Micobatteri, Dipartimento di Scienze ed Oncologia Umana, Sezione di Igiene, Policlinico-Bari

I Clinica Malattie Infettive, Policlinico-Bari

2 Unità di Genomica Dipartimento Biotecnologie Metaponto-Agrobios, Laboratorio Osp. Madonna delle Grazie, Matera

3 Laboratorio Microbiologia Osp. Madonna delle Grazie, Matera

Key Words: Nocardia, Liquor, Kidney transplant recipient, Sequencing of hsp65 gene.

Meningo-encefalite diffusa da Nocardia farcinica in giovane paziente immunocompromesso trapiantato di reni: identificazione mediante sequenziamento del gene hsp65 usato per l'identificazione dei micobatteri

\section{SUMMARY}

Genus Nocardia belongs to aerobic Actinomycetes, a wide group of polymorphous Gram+, fixed and acapsusalated, branching, partially acid-fast bacilli. Nocardia organism are ubiquitous, soil-borne actinomycetes that usually infect humans as a result of the inhalation of airborne bacilli or traumatic inoculation. Nocardiosis is a rare opportunistic disease that affects mainly patients with impaired cellmediated immunity, such as those with acquired immunodeficiency syndrome (AIDS) or transplant recipients, patients with pulmonary disease, haematological malignancies etc.

Pulmonary disease is the most common presentation in immounosuppressed patients. Nocardial organism have a tendency to disseminate hematogenously from the primary site of infection. The central nervous system (CNS) is one of the most frequent sites of dissemination. Herein we describe a rare and fatal case of diffuse meningo-encephalytis due to Nocardia farcinica in a young patient kidney transplant recipient. Nocardia has been isolated from Cerebrospinal fluid in a micobacteriology laboratory with identification of the strain using sequencing of hsp65 gene.

The diagnosis can be challenging, as signs and symptoms are not specific and a high degree of clinical suspicion is required. Identification of Nocardia farcinica is important because of its aggressiveness, its tendency to disseminate, and its resistance to antibiotics.

Susceptibilities to 10 antimicrobial agents were determined by E-test. The isolate was resistant to Gentamicin, Clarithromycin, Doxycicline, Cefotaxime and susceptible to Amikacin, Amoxicillin clavulanate, Imipenem, Ciprofloxacin, Linezolid and Trimethoprim-Sulfamethoxazole. The susceptibility profile was favourable since, in North Italy, the strains are generally resistant to Trimethoprim-Sulfamethoxazole.

\section{INTRODUZIONE}

La nocardiosi è una rara infezione opportunistica, che, in Europa e negli Stati Uniti, può avere un decorso più acuto e aggressivo nei pazienti con deficit dell'immunità cellulomediata. In particolare quella che si realizza in trapiantati di organo, in pazienti con neoplasie linforeticolari e in tutte quelle forme di immunodepressione con alterazione dell'immunità cellulare (HIV- positivi, affetti da patologie autoimmuni, pazienti in terapia con farmaci citostatici).

La localizzazione polmonare è quella più comune, favorita dal fatto che il contagio avviene prevalentemente per via aerea. Nelle nocardiosi la diffusione al sistema nervoso centrale (SNC) si verifica nel 20-40\% dei casi e si estrinseca clinicamente, per lo più, come ascesso cerebrale $(3,10,14)$.In Asia invece è una patologia soprattutto cutanea, anche nell'immunocompetente.

Qui di seguito descriviamo un raro caso di meningo-encefalite diffusa da Nocardia farcinica in giovane paziente trapiantato di reni. Il germe è stato isolato da liquor in laboratorio di micobatteriologia, con identificazione del ceppo mediante sequenziamento del gene hsp65.

\section{CASO CLINICO}

Nel settembre 2009 viene ricoverato presso la Clinica delle Malattie Infettive del Policlinico di Bari un paziente di 28 anni, per febbre ad andamento remittente insorta una settimana prima e segni di rigor nucalis riscontrati nel giorno del ricovero. Il paziente era stato trapiantato di rene nel 2000 per insufficienza renale cronica terminale da nefropatia ad IgA, trattata, fino all'epoca del trapianto, con emodialisi periodica. Oltre alla nefropatia, in anamnesi il paziente presentava, una epatopatia cronica evoluta con episodio di scompenso ascitico nel 2004 e diabete mellito. Era stato inoltre splenectomizzato nel 2008 per incidente della strada. Il paziente eseguiva terapia immunosoppressiva con prednisone per os 50 $\mathrm{mg} /$ die, tacrolimus $2 \mathrm{mg} /$ die e acido micofenolico $2 \mathrm{mg} /$ die, terapia antipertensiva con irbesartan $300 \mathrm{mg} / \mathrm{die}$, ramipril 10 $\mathrm{mg} /$ die, atenololo $50 \mathrm{mg} /$ die, terapia diuretica con furosemide $100 \mathrm{mg} /$ die, canrenoato di potassio $100 \mathrm{mg} /$ die, terapia antidislipidemica con ezetimibe $10 \mathrm{mg}$ associato a simvastatina $20 \mathrm{mg}$, terapia antidiabetica con repaglinide $6 \mathrm{mg} / \mathrm{die}$ e terapia antiulcera gastrica con lansoprazolo $60 \mathrm{mg} /$ die.

$\mathrm{Al}$ ricovero il paziente presenta febbre elevata, cefalea e violente rachialgie. Obiettivamente si riscontra rigor nucalis, positività al segno di Lasegue e sensorio lievemente obnubilato. Una risonanza magnetica nucleare del cranio eseguita il giorno del ricovero evidenzia una diffusa alterazione di segnale di tipo flogistico a carico del ponte e del mesencefalo bilateralmente, del talamo di sinistra, delle cisterne basali, peripontina e perimesencefalica e dell'ependima che risulta impregnato dopo somministrazione di mezzo di contrasto. Una prima rachicentesi evidenzia liquor di aspetto torbido, leucorrachia (2.575 leucociti/mm3, con il 98\% di linfociti), lieve iperproteinorrachia (128 mg/dl), ipoglucorrachia (25 $\mathrm{mg} / \mathrm{dl})$ e iperlattatemia $(79 \mathrm{mg} / \mathrm{dl})$.

Il paziente inizia subito dopo la rachicentesi trattamento empirico con ampicillina/sulbactam endovena, $12 \mathrm{~g} /$ die e vancomicina endovena $2 \mathrm{~g} / \mathrm{die}$, associati a terapia antiedemigena con mannitolo. Il trattamento non determina alcun miglioramento del quadro clinico nei giorni successivi. Le indagini colturali per germi comuni eseguite su liquor e le emocolture risultano negative.

In $4^{\circ}$ giornata, una PCR per CMV su liquor mostra positività con 323 genomi/ml, per cui viene aggiunto in terapia gan-

\section{Corresponding author: Danila Costa}

U.O.C. Igiene; Azienda Ospedaliera Policlinico Bari

Tel.: 0805593300 - Fax: 0805478472

E-mail: costad7@libero.it 
ciclovir endovena $500 \mathrm{mg} /$ die, anche in questo caso senza alcun miglioramento clinico.

In $7^{\circ}$ giornata si riscontra una parziale positività su liquor germi acido-alcol resistenti per cui, in attesa degli esami colturali, la terapia in atto viene sostituita con una terapia antitubercolare endovenosa a base di rifampicina $600 \mathrm{mg}$, isoniazide $300 \mathrm{mg}$ ed etambutolo $1.2 \mathrm{~g}$.

In $11^{\circ}$ giornata il paziente sviluppa idrocefalo tetraventricolare evidenziato alla TAC e viene posizionato drenaggio ventricolare esterno mediante catetere di derivazione a partenza dalla regione frontale destra e termine nel corno frontale omolaterale.

In $12^{\circ}$ giornata, non riscontrandosi alcun miglioramento alla terapia antitubercolare in atto, si aggiunge empiricamente streptomicina $1 \mathrm{~g} /$ die ev e levofloxacina $500 \mathrm{mg}$ x $2 \mathrm{ev}$.

Nonostante le modifiche terapeutiche effettuate, la liquorchimica, ripetuta in $4^{\circ}, 7^{\circ}, 11^{\circ}$ e $13^{\circ}$ giornata, si mantiene sostanzialmente sovrapponibile, la febbre persiste e le condizioni cliniche del paziente peggiorano progressivamente con persistenza di febbre, ad andamento di tipo remittente. In $14^{\circ}$ giornata di ricovero il paziente decede.

Gli esiti delle indagini di laboratorio, pervenuti subito dopo il decesso del paziente hanno permesso di identificare in tutti i campioni di liquor la presenza di Nocardia farcinica.

\section{MERIALI E METODI}

Il campione di liquor è stato messo in coltura (senza decontaminazione), nei terreni usati per la diagnostica micobatterica: L-J medium, L-J+TCH, Middlebrook 7H10 agar, terreno liquido 7H9 Bactec MGIT 960.

Il terreno liquido ha mostrato sviluppo di colonie dopo 6 giorni di incubazione, mentre i terreni solidi dopo 9-10 giorni con sviluppo di colonie giallo paglierino con morfologia prima liscia poi rugosa. La microscopia (sec. Ziehl-Neelsen opp. Kinyoun) delle colonie in coltura pura, ha messo in evidenza batteri coccoidi che avevano perso la loro iniziale acido-alcool resistenza parziale, ovviamente Gram+. Sono state così effettuate sottocolture in agar Sabouraud, agar-sangue e Thayer-Martin: le colonie mostravano sui diversi terreni uno spiccato polimorfismo sia riguardo il colore (dal giallo all'arancio) sia riguardo la morfologia, prima liscia e poi rugosa con presenza di ife aeree. Dopo aver osservato sviluppo di colonie a $45^{\circ} \mathrm{C}$ e l'opacizzazione del 7H10 agar (5), il ceppo è stato inviato al laboratorio di biologia molecolare del centro diagnostico “X-life”, dell’ospedale "Madonna delle Grazie” di Matera (Basilicata) per l'identificazione tramite sequenziamento del DNA del gene hsp65 che normalmente viene utilizzato per identificare NTM (micobatteri non tubercolari) $(2,15,16)$.

Indagine gene hsp65 su lisato

a) amplificazione del gene hsp65: La reazione di amplificazione è condotta direttamente su lisato cellulare. La colonia da analizzare è immersa in $200 \mu \mathrm{l}$ di $\mathrm{H}_{2} 0$ sterile, riscaldata per 5' a $100^{\circ} \mathrm{C}$; subito dopo raffreddata velocemente in $\mathrm{H}_{2} \mathrm{O}$ fredda. Lo shock termico, dovuto all'immersione in $\mathrm{H}_{2} \mathrm{O}$ fredda, provoca la rottura della parete cellulare con la conseguente uscita del DNA. Il lisato così ottenuto è centrifugato e si utilizzano $10 \mu \mathrm{l}$ del surnatante per effettuare la reazione di PCR. La reazione di amplificazione è effettuata secondo le condizioni suggerite da Talenti, et al (17) con l'impiego dei primers TB11 e TB12.

b) analisi di sequenza: L'amplicone di circa 440 bp del gene hsp65, è sequenziato utilizzando il kit Big Dye Terminator Cycle Sequencing (vers. 3.1, Applied Biosystems). Le reazioni di sequenza sono state sottoposte ad elettroforesi capillare e le sequenze sono state rilevate ed analizzate su un sequenziatore capillare ABI Model 3130 (Applied Biosystems). Le analisi di sequenza sono state condotte utilizzando il programma Sequencher (GenesCodes Corporation).

L'identità del ceppo ottenuto è stata confermata tramite la comparazione delle sequenze parziali del gene hsp65 con le sequenze disponibili nelle banche dati del NCBI (http: //www.ncbi.nlm.nih.gov) e EBI (http://www.ebi.ac.uk ). È stata analizzata la parte variabile del gene hsp65 ed è stato ottenuto un grado di concordanza del 99\% con la sequenza dell'hsp65 di Nocardia farcinica (Figura I).

Test di sensibilità agli antibiotici: è stata valutata la sensibilità a dieci antibiotici mediante E-test (PDMEpsilometer, AB BIODISK, Solna, Sweden). Il ceppo di Nocardia farcinica isolato ha mostrato sensibilità nei confronti dei seguenti farmaci: Amikacina, Amoxi/Clav, Imipenem, Ciprofloxacina, Linezolid, Trim/Sulfa ed è risultato resistente a: Claritromicina, Gentamicina, Doxyciclina e Cefotaxime (Tabella 1 e Figura II).

Le colonie di Nocardia sono difficilmente stemperabili, l'inoculo è stato preparato vortexando a secco le colonie con biglie di vetro (3 $\mathrm{mm}$ ), aggiungendo $\mathrm{MH}$ brodo fino al raggiungimento di una torbidità pari a $1.0 \mathrm{McF}$ arland $(1,7,8,9)$. La sospensione è stata inoculata in agar medium MuellerHinton $+5 \%$ di sangue di montone.

Le piastre sono state incubate a $35^{\circ} \mathrm{C}$ per $72 \mathrm{~h}$.

\section{DISCUSSIONE}

La rapidità dell'evoluzione clinica del caso e la rarità della patologia, purtroppo non ha consentito di instaurare per tempo un'adeguata terapia antibiotica. Indubbiamente l'immunodeficenza legata sia alla terapia antirigetto, sia allo stato di asplenia ha giocato un ruolo importante nel determinare un quadro atipico e così rapidamente evolutivo. Tuttavia l'individuazione, quantunque post-mortem, dell'agente patogeno, ci consente numerose considerazioni $(7,11)$.

L’incidenza di nocardiosi è stimata molta bassa (circa 5001000 nuovi casi all'anno negli U.S.A.); questo dato rappresenta probabilmente una sottostima del fenomeno, in ragione di vari motivi:

1) Difficoltà di diagnosi, che viene compiuta in media molto tardivamente o post-mortem;

2) La necessità di laboratori di "riferimento" per l'isolamento e l'identificazione del patogeno;

3) Bassa sensibilità e specificità degli esami non invasivi comunemente utilizzati nella diagnostica microbiologica;

4) Scarsa propensione dei clinici a considerare questo patogeno nel ventaglio delle possibili eziologie microbiche.

La maggior parte delle nocardiosi umane sono sostenute da Nocardia asteroides complex, responsabile dell' $80 \%$ circa delle forme cliniche invasive non cutanee e che interessano il SNC. La Nocardia farcinica è la specie più importante del complesso, per il suo potenziale patogeno, per la tendenza a disseminare dai siti di infezione primaria e per la resistenza agli antibiotici $(3,12,14)$.

Nocardia brasiliensis è invece il più importante agente delle forme ad estrinsecazione cutanea o linfocutanea, anche se può essere responsabile di forme sistemiche o, occasionalmente, neurologiche (2).

Il genere Nocardia appartiene agli Actinomiceti aerobi, un ampio gruppo di batteri polimorfi Gram+, immobili e acapsulati. Si tratta di microrganismi ubiquitari, il loro habitat si ritrova nel suolo, nelle acque e possono infettare oltre all'uomo, piante e animali.

I membri di questo gruppo: Mycobacterium, Corynebacterium, Nocardia, Rhodococcus, Gordonia, TsuKamurella e Tropheryma whippeli (Whipple's desease) pur non essendo strettamente correlati tra di loro dal punto di vista filogenetico, hanno tra i costituenti della loro parete batterica gli acidi micolici responsabili di una variabile acido resistenza. Questo sottolinea l'importanza di accurata diagnostica differenziale $(2,3,11)$.

Le Nocardie all'esame microscopico mostrano filamenti che tendono a frammentarsi in forme bacillari e coccoidi, le colonie crescono bene sia su terreni per micobatteri e per miceti, sia su agar-sangue.

La genetica molecolare, ci permette di identificare diverse specie di Nocardia, potendo così superare le tecniche stan- 
dard di laboratorio piuttosto limitate nella loro abilità a differenziare questi microrganismi $(2,15,16)$. Il gene hsp65, responsabile della sintesi dell'antigene 65K, è altamente conservato, è una heat shock protein che viene sintetizzata in particolari condizioni di stress cellulare, è presente in tutte le specie di micobatteri e di alcune specie di Actinomiceti, come le Nocardie, ed è caratterizzata da un alto grado di variabilità.

È importante sospettare la presenza di questo agente, anche con presentazioni atipiche come quella descritta, in pazienti immunodepressi, al fine di avviare tempestivamente l'opportuno iter diagnostico e terapeutico $(4,6,19)$.

Tabella I. Test di sensibilità agli antibiotici eseguito mediante E-test sul ceppo di Nocardia farcinica isolato

\begin{tabular}{llll}
\hline Antibiotico testato & MIC $(\mu \mathrm{g} / \mathrm{ml})$ & \multicolumn{2}{l}{ Interpretazione $\mathrm{MIC}(\mu \mathrm{g} / \mathrm{ml})$} \\
\hline Amikacina $(\mathrm{AK})$ & $\mathrm{I})$ & Sensibile & $\leq 8$ \\
\hline Amoxi/Clav $(\mathrm{XL})$ & 2 & Sensibile & $\leq 8 / 4$ \\
\hline Imipenem (IP) & 3 & Sensibile & $\leq 4$ \\
\hline Ciprofloxacina (CL) & 0.094 & Sensibile & $\leq \mathrm{I}$ \\
\hline Linezolid (LZ) & 2 & Sensibile & $\leq 8$ \\
\hline Trim/Sulfa (TS) & 1.5 & Sensibile & $\leq 2$ \\
\hline Claritromicina (CH) & 16 & Resistente & $\geq 8$ \\
\hline Gentamicina (GM) & 32 & Resistente & $\geq 16$ \\
\hline Doxyciclina (DC) & 64 & Resistente & $\geq 8$ \\
\hline Cefotaxime (CT) & 128 & Resistente & $\geq 64$ \\
\hline
\end{tabular}

>EM_PRO:AY756523;AY756523 Nocardia farcinica strain DSM 4366565 kDa heat shock protein (hsp65) gene, partial cds. Length $=44$ I

Plus Strand HSPs:

Score $=2096$ (320.5 bits), Expect $=2.1 \mathrm{e}-86, \mathrm{P}=2.1 \mathrm{e}-86$

Identities $=420 / 421$ (99\%), Positives = 420/42I (99\%), Strand $=$ Plus $/$ Plus

Query: I GTGTGTCCATCGCCAAGGAGATCGAGCTGGAGGATCCCTACGAGAAGATCGGCGCCGAGC 60 ||||||||||||||||||||||||||||||||||||||||||||||||||||||||||||||

Sbjct: I I GTGTGTCCATCGCCAAGGAGATCGAGCTGGAGGATCCCTACGAGAAGATCGGCGCCGAGC 70

Query: 61 TGGTCAAGGAAGTCGCCAAGAAGACCGACGACGTCGCGGGCGACGGCACCACCACCGCCA 120 ||||||||||||||||||||||||||||||||||||||||||||||||||||||||||||||

Sbjct: $\quad 71$ TGGTCAAGGAAGTCGCCAAGAAGACCGACGACGTCGCGGGCGACGGCACCACCACCGCCA I30

Query: $\quad$ |2| CCGTGCTCGCCCAGGCGCTGGTGCGCGAGGGTCTGCGCAACGTGGCCGCGGGCGCGAACC I80 |||||||||||||||||||||||||||||||||||||||||||||||||||||||||||

Sbjct: $\quad|3|$ CCGTGCTCGCCCAGGCGCTGGTGCGCGAGGGTCTGCGCAACGTGGCCGCGGGCGCGAACC 190

Query: I8I CGCTGGGCCTCAAGCGCGGCATCGAGAAGGCCGTCGAGGCCGTGACCGCCAAGCTGCTCG 240 |||||||||||||||||||||||||||||||||||||||||||||||||||||||||||||||

Sbjct: $\quad 191$ CGCTGGGCCTCAAGCGCGGCATCGAGAAGGCCGTCGAGGCCGTGACCGCCAAGCTGCTCG 250

Query: 24I ACACCGCCAAGGAGGTCGAGACCAAGGAGCAGATCGCCGCCACCGCGGGCATCTCCGCGG 300 |||||||||||||||||||||||||||||||||||||||||||||||||||||||||||||

Sbjct: 25I ACACCGCCAAGGAGGTCGAGACCAAGGAGCAGATCGCCGCCACCGCGGGCATCTCCGCGG 310

Query: 301 GCGACGCGTCCATCGGTGAGCTGATCGCCGAGGCCATGGACAAGGTCGGCAAGGAAGGCG 360 ||||||||||||||||||||||||||||||||||||||||||||||||||||||||||||||

Sbjct: $\quad 311$ GCGACGCGTCCATCGGTGAGCTGATCGCCGAGGCCATGGACAAGGTCGGCAAGGAAGGCG 370

Query: $\quad 36 I$ TCATCACCGTCGAGGAGAGCAACACCTTCGGCCTCCAGCTGGAGCTGACCGAGGGTATGC 420 ||||||||||||||||||||||||||||||||||||||| ||||||||||||||||||||||||

Sbjct: $\quad 371$ TCATCACCGTCGAGGAGAGCAACACCTTCGGCCTCCATCTGGAGCTGACCGAGGGTATGC 430

Query: 421 G 42I

Sbjct: $\quad 431$ G 43 I

Figura I. Analisi di sequenza del gene hsp65

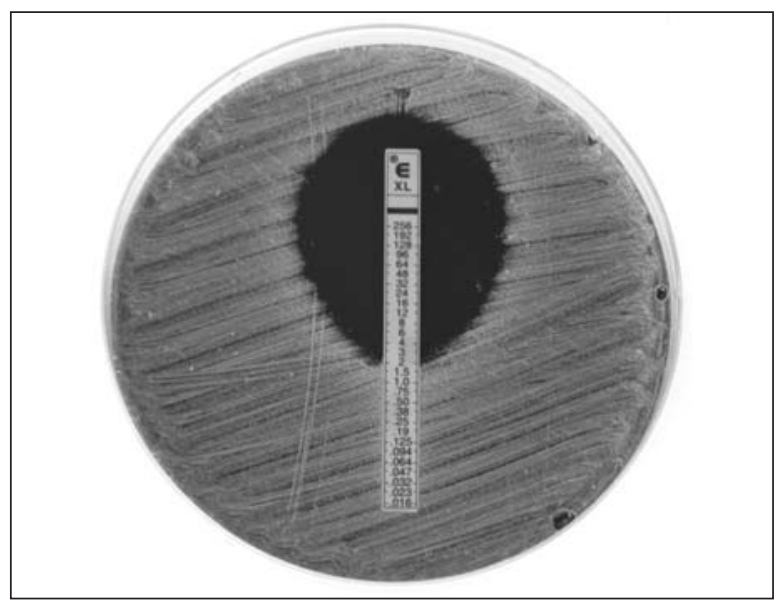

Figura II. Immagine dell'E-test per amoxicillina/acido clavulanico (XL). 


\section{BIBLIOGRAFIA}

1. Biehle JB, Cavalieri SJ, Saubolle MA, et al. Comparative evaluation of the E test for susceptibility testing of Nocardia species. Diagn Microb infect Dis 1994; 19: 101-10.

2. Brown-Elliot BA, Brown JM, Conville PS, Wallace Jr RJ. Clinical and Laboratory Features of the Nocardia spp. Based on Current Molecular Taxonomy. Clinical Microbiol Rev 2006; 19: 259-82.

3. Carosi G, Pauluzzi S. Malattie Infettive. Piccin Nuova Libraria Spa, 215-7.

4. Chih-Cheng Lai, Li-Na Lee, Lee-jene Teng, et al. Disseminated Nocardia farcinica infection in a uraemia patient with idiopathic thrombocytopenia purpura receiving steroid therapy. $J$ Med Microbiol 2005; 54: 1107-10.

5. De La Iglesia P, Viejo G, Gomez B, et al. Fatal Pulmonary Nocardia farcinica Infection. J Clin Microbiol 2002; 40: 1098-9.

6. Dias M, Antony B, Pinto H. Spectrum of Nocardiosis. A report of three cases. Jour of Clin and Diagn Res 2009; 3: 1682-4.

7. Falcone M, Pompeo ME, Fabi F, Venditti M. Efficacia di linezolid nel trattamento della nocardiosi: descrizione di un caso e rassegna della letteratura. Le infezioni in medicina 2008; 2: 94-8.

8. Farina C, Boiron P, Goglio A, Provost F. Human nocardiosis in northern Italy from 1982 to 1992. Northern Italy Collaborative Group on Nocardiosis. Scand J Infect Dis. 1995; 27: 23-7.

9. Glupczynski Y, Berhin C, Janssens M, Wauters G. Determination of antimicrobial susceptibility patterns of Nocardia spp. From clinical specimens by E test. Clin Microbiol Infect 2006; 12: 905-12.

10. Jimenez-Galanes Marchan S, Meneu Dìaz JC, Caso Maestro O, et al.
Disseminated nocardiosis: a rare infectious complication following nonheart-beating donor liver transplantation. Transplant Proc 2009; 41: 2495-7.

11. Lerner PI. Nocardiosis. Clin Infect Dis 1996; 22: 891.

12. Malincarne L, Marroni M, Farina C, Camanni G, et al. Primary brain abscess with Nocardia farcinica in an immunocompetent patient. Clin Neur and Neuros 2002;104: 132-5.

13. Murray PR, Niles AC, Heeren RL. Modified thayer-martin medium for recovery of Nocardia Species from contaminated specimens. Jour of Clin Microb, 1988; 26: 1219-20.

14. Nampoory MRN, Khan ZU, Johny KV, et al. Nocardiosis in renal transplant recipients in Kuwait. Nephrol Dial Trans 1996; 11: 1134-8.

15. Po-Ling Chang, Wen-Shyang Hsieh, Chia-Lien Chiang, Marion J. Tuohy, et al. The hsp65 gene patterns of less common Mycobacterium and Nocardia spp. By polymerase chain reaction-restriction fragment length polymorphism analysis with capillary electrophoresis. Diagn Microb and Infect Dis 2007; 58: 315-23.

16. Rodriguez-Nava V, Couble A, Devulder G, et al. Use of PCR-Restriction enzyme pattern analysis and sequencing database for hsp65 gene-based identification of Nocardia Species. Jour of Clin Microb 2006; 44: 536-46. 17. Talenti, et al. J Clin Microbiol 1993; 31: 406-9.

18. Torres OH, Domingo O, Pericas R, et al. Infection caused by Nocardia farcinica: case report and review. Eur J Clin Microbiol Infect Dis 2000; 19: 205-12.

19. Viganò SM, Edefonti A, Ferraresso M, et al. Successful medical treatment of multiple brain abscesses due to Nocardia farcinica in a paediatric renal transplant recipient. Pediatr Nephrol 2005; 20: 1186-8. 